\title{
Murine models provide insight to the development of non-alcoholic fatty liver disease
}

\author{
D. T. Reid and B. Eksteen* \\ Snyder Institute for Chronic Diseases, Cumming School of Medicine, University of Calgary, Calgary, Alberta, Canada
}

\section{Abstract}

Associated with the obesity epidemic, non-alcoholic fatty liver disease (NAFLD) has become the leading liver disease in North America. Approximately $30 \%$ of patients with NAFLD may develop non-alcoholic steatohepatitis (NASH) that can lead to cirrhosis and hepatocellular carcinoma (HCC). Frequently animal models are used to help identify underlying factors contributing to NAFLD including insulin resistance, dysregulated lipid metabolism and mitochondrial stress. However, studying the inflammatory, progressive nature of NASH in the context of obesity has proven to be a challenge in mice. Although the development of effective treatment strategies for NAFLD and NASH is gaining momentum, the field is hindered by a lack of a concise animal model that reflects the development of liver disease during obesity and the metabolic syndrome. Therefore, selecting an animal model to study NAFLD or NASH must be done carefully to ensure the optimal application. The most widely used animal models have been reviewed highlighting their advantages and disadvantages to studying NAFLD and NASH specifically in the context of obesity.

\section{Key words: Obesity: Non-alcoholic fatty liver: Non-alcoholic steatohepatitis: Animal models}

\section{Introduction}

In less than 50 years obesity has become a worldwide epidemic and is estimated to affect over one billion individuals ${ }^{(1)}$. One of the most concerning aspects related to obesity is the increased risk of developing the metabolic syndrome and associated co-morbidities ${ }^{(2)}$. Currently the leading cause of liver disease in North America, non-alcoholic fatty liver disease (NAFLD) is considered the hepatic manifestation of the metabolic syndrome and is characterised as an increase in intrahepatic fat that may be accompanied by inflammation in the absence of alcohol abuse ${ }^{(3,4)}$

NAFLD includes a spectrum of liver diseases that includes simple steatosis as well as the progression of non-alcoholic steatohepatitis (NASH) that can develop into cirrhosis and hepatocellular carcinoma (HCC) ${ }^{(5,6)}$ (Fig. 1). Although it is estimated that up to $90 \%$ of individuals with obesity (BMI $>30$ $\mathrm{kg} / \mathrm{m}^{2}$ ) may have hepatic steatosis, this condition represents a relatively benign and asymptomatic accumulation of intrahepatic fat ${ }^{(7)}$. However, approximately $30 \%$ of patients will develop steatohepatitis characterised by inflammation leading to hepatocyte damage and potentially fibrosis ${ }^{(5)}$. Given time, NASH can progress to liver cirrhosis and $\mathrm{HCC}^{(6,8)}$. While pharmacological treatments are being actively studied they are in the early stages of development; therefore current treatment strategies for NASH are limited to intensive dietary and lifestyle modification ${ }^{(9,10)}$. Due to the severe complications associated with NASH, identification of the underlying mechanisms driving its pathogenesis is required.

The factors contributing to the development of NAFLD are under extensive study. NAFLD is associated with obesity, insulin resistance, diabetes, hyperlipidaemia and increased risk of CVD and cancer ${ }^{(6,11)}$. The accumulation of central adiposity along with insulin resistance has been linked to the development of liver steatosis and probably contributes to the further progression to $\mathrm{NASH}^{(12,13)}$. Genetic susceptibility and environmental factors such as diet have also been implicated in disease progression of $\mathrm{NASH}^{(13)}$. Although excessive adiposity and the metabolic syndrome are linked to the majority of cases of NAFLD, steatohepatitis has also been reported as a bystander effect due to pharmacological therapies most commonly associated with oxidative stress and aberrant changes in lipid metabolism ${ }^{(14)}$. Individuals receiving total parenteral nutrition (TPN) are also at increased risk of developing $\mathrm{NASH}^{(14,15)}$. Recently it was shown that patients with chronic hepatitis C carrying the PNPLA3 rs738409 G variant is associated with more severe steatosis and steatohepatitis in the absence of obesity ${ }^{(16)}$. Caution should be taken when interpreting results based on the characterisation of non-obese patients with steatosis or steatohepatitis as many studies use BMI for the classification of obesity; however, this has been shown to underestimate the prevalence of obesity in a number of different racial/ethnic populations ${ }^{(17-19)}$.

Abbreviations: ALT, alanine aminotransferase; HCC, hepatocellular carcinoma; HFD, high-fat diet; MCD, methionine- and choline-deficient; NAFLD, non-alcoholic fatty liver disease; NASH, non-alcoholic steatohepatitis; TPN, total parenteral nutrition.

* Corresponding author: Dr Bertus Eksteen, fax +1 403210 9146, email b.eksteen@ucalgary.ca 

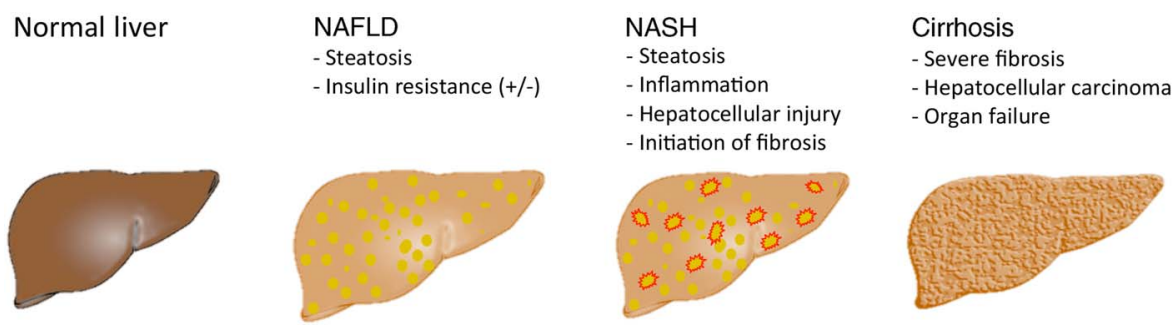

Fig. 1. A current model demonstrating the progression of non-alcoholic fatty liver disease (NAFLD) in humans. While animal models can recapitulate certain aspects of NAFLD, non-alcoholic steatohepatitis (NASH) and even fibrosis, not one model exists that covers all stages of the liver disease. Further development of animal models is needed to continue the study of disease mechanisms and evaluation of possible treatment strategies.

The use of animal models has proven to be a useful tool to provide insight into the development of NAFLD and progression of NASH. However, research in this field has been hampered by the lack of an animal model that can fully recapitulate the disease phenotype in a consistent and timely manner. Due to the number of factors that are associated with the progression of NAFLD and still unclear pathogenesis, rodent models must be carefully chosen to answer the research question. Highlighting this fact is a recent publication directly comparing two widely used dietary murine models of NAFLD demonstrating the critical impact of diet on the pathogenesis of these diseases ${ }^{(20)}$. In addition, innovative murine models are still needed to facilitate evaluation of novel treatments for NAFLD and NASH.

\section{Pathogenesis of non-alcoholic fatty liver disease}

We have learned a considerable amount about NAFLD since it was first described over three decades ago ${ }^{(21)}$. The development of NAFLD in patients has been closely linked to adipose tissue insulin resistance ${ }^{(10)}$. During obesity, loss of peripheral insulin sensitivity may lead to the aberrant release of NEFA from adipose tissue as well as increased de novo lipogenesis promoting hepatic steatosis ${ }^{(22)}$. It is thought that an influx of NEFA from adipose tissue stores may contribute up to $80 \%$ of intrahepatic fat ${ }^{(23)}$. Diets higher in saturated fat and cholesterol have been linked to patients with NAFLD and may influence the development of hepatic insulin resistance ${ }^{(23,24)}$. In addition, levels of proinflammatory cytokines including TNF- $\alpha$ and IL-6 as well as elevated levels of leptin and reduced adiponectin have been detected during NAFLD ${ }^{(10,25,26)}$. Recent advances in the understanding of autophagy put into question the exact role of insulin resistance during NAFLD with reports suggesting the accumulation of excess lipid within the liver may in fact initiate insulin resistance ${ }^{(27)}$. Considerable research is required to further understand the contribution of autophagy to the pathogenesis of obesity, the metabolic syndrome and NAFLD ${ }^{(28)}$.

The diagnosis of NAFLD often occurs in asymptomatic patients following the detection of elevated serum aminotransferases and/or ultrasound findings of steatosis during routine screening ${ }^{(29)}$. A positive diagnosis of NAFLD usually requires the exclusion of all other causes of liver steatosis including viral infection and autoimmunity as well as the assessment of liver function, lipid profiles and insulin sensitivity measured by the homeostatic model assessment of insulin resistance (HOMA-IR) index ${ }^{(30)}$. Alcohol abuse causing alcoholic liver disease must also be separately identified ${ }^{(21)}$. Interestingly, moderate ethanol consumption $(2.5 \mathrm{~g} / \mathrm{kg}$ body weight per $\mathrm{d})$ in leptin-deficient $(o b / o b)$ mice was recently associated with reduced steatosis and improved liver function possibly due to increased adiponectin levels and enhanced activation of the sirtuin 1 (SIRT1) pathway and Akt phosphorylation $^{(31)}$. This demonstrates that the degree of alcohol consumption and NAFLD should be considered although this is currently beyond the scope of the present review. Liver biopsy remains the 'gold standard' for diagnosis; however, the use of biomarkers is currently being developed as a less invasive diagnostic tool ${ }^{(32)}$.

Development of non-alcoholic steatohepatitis from obesity and non-alcoholic fatty liver disease

The development of NASH was previously associated with a 'two-hit' model where liver steatosis is accompanied by the metabolic syndrome that progresses into hepatitis following additional stresses ${ }^{(33)}$. However, this theory has now been extended to include multiple factors that are considered to promote the development of inflammation and fibrosis including increased mitochondrial dysfunction, endoplasmic reticulum stress, changes in lipid oxidation and increased endotoxin levels in the liver leading to immune cell activation and infiltration contributing to a heightened proinflammatory microenvironment leading to hepatocyte cell stress and death $^{(34-38)}$.

The histopathology of NASH includes evidence of steatosis, both macro- and microvesicular as well as ballooning hepatocytes, lobular inflammation and the development of pericellular fibrosis ${ }^{(39)}$. Elevated aminotransferase levels including alanine aminotransferase (ALT) and aspartate aminotransferase may signal changes in liver biochemistry but are inconsistent whereas liver biopsy remains the 'gold standard' diagnostic procedure for $\mathrm{NASH}^{(30)}$. Given the heterogeneity inherent to the development of NASH, treatment of this disease poses a challenge. Selecting an appropriate model that captures the underlying chronic, low-grade inflammation due to the metabolic syndrome along with the active inflammation process is key. Although a single animal model has yet to capture the complete human disease pathology a number of models are able to recapitulate certain disease pathologies (Table 1). 
Murine models with increased genetic susceptibility to non-alcoholic fatty liver disease

When choosing an animal model to study obesity, type 2 diabetes and fatty liver disease the genotype $\mathrm{x}$ environment interactions should be considered ${ }^{(40-42)}$. A distinct difference was detected when comparing the disease susceptibility to high-fat diet (HFD) treatment between two commonly used mouse strains $\mathrm{C} 57 \mathrm{BL} / 6 \mathrm{~J}$ and $\mathrm{BALB} / \mathrm{c}^{(40)}$. Following 23 weeks of HFD $\mathrm{C} 57 \mathrm{BL} / 6 \mathrm{~J}$ mice gained more weight and had higher adiposity compared with BALB/c mice ${ }^{(40)}$. Analysis of gene transcription in the liver between the mouse strains demonstrated that each strain differentially regulated genes associated with fatty acid uptake and efflux when fed HFD affording increased disease susceptibility to $\mathrm{C} 57 \mathrm{BL} / 6 \mathrm{~J}$ mice and relative resistance to BALB/C mice fed a HFD ${ }^{(40)}$. To further support this finding, 8 weeks of HFD treatment in C57BL/6J, 129X1/SvJ, DBA/2 and FVB/N mice resulted in similar body-weight gain and impaired glucose tolerance whereas BALB/c mice had normal glucose tolerance and resisted hepatic lipid accumulation ${ }^{(41)}$. It has been suggested that while $\mathrm{C} 57 \mathrm{BL} / 6 \mathrm{~J}$ mice are the most susceptible to dietinduced obesity, BALB/C mice might be a good model of 'healthy obese humans' since BALB/c mice have lower hepatic lipid accumulation and normal glucose tolerance ${ }^{(41)}$. Within the C57 sub-strains, C57BLKS/J mice have been reported to gain less weight over 12 weeks of HFD treatment and have improved glucose tolerance compared with $\mathrm{C} 57 \mathrm{BL} / 6 \mathrm{~J}$ mice although the strains share $70 \%$ genetic homology ${ }^{(43)}$. Genetic differences between $\mathrm{C} 57 \mathrm{Bl} / 6 \mathrm{~N}$ and $\mathrm{C} 57 \mathrm{BL} / 6 \mathrm{~J}$ mice have also been linked to differences in disease susceptibility in HFD-fed mice ${ }^{(44,45)}$.

Numerous genetic models of both NAFLD and NASH have been recently described ${ }^{(46)}$. These models may develop obesity, impaired insulin sensitivity, glucose intolerance and hepatic steatosis over time independent of dietary challenge ${ }^{(46)}$. In other cases dietary manipulation is required to expose the development of the metabolic syndrome ${ }^{(46)}$. Some of the more widely used models have been highlighted in greater depth below.

\section{$\mathrm{LDLr}^{-/-}$}

Often mice with a genetic alteration will be treated with a HFD to exacerbate an underlying phenotype. This is particularly important for the LDL receptor-deficient mouse. Due to a lack of LDL receptors and an inability to clear intermediate-density lipoproteins and LDL these mice develop hypercholesterolaemia and hypertriacylglycerolaemia when fed a $\operatorname{HFD}^{(47)}$. One of the mechanisms that has been proposed for the development of the insulin-resistant phenotype observed in the $\mathrm{LDLr}^{-/}$mouse is due to a lack of $\beta$-cell hypertrophy to compensate for reduced insulin release ${ }^{(47)}$. Treatment of a high-fat high-cholesterol diet in $\mathrm{LDLr}^{-/}$mice for 12 weeks leads to hyperlipidaemia as well as glucose and insulin intolerance ${ }^{(48)}$. $L D L r^{-/}$mice fed a Western diet supplemented with olive oil for 16 weeks resulted in a robust steatohepatitis phenotype including elevated ALT levels, inflammation and the development of early fibrosis ${ }^{(49)}$. A high-fat, high-carbohydrate diet with added cholesterol has also been shown to induce a NASH-like phenotype in $\mathrm{LDLr}^{-/}$mice after 24 weeks of treatment ${ }^{(50)}$. 
(a)

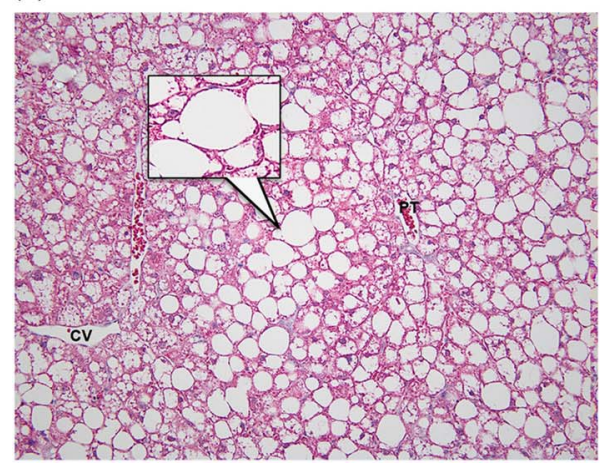

(b)

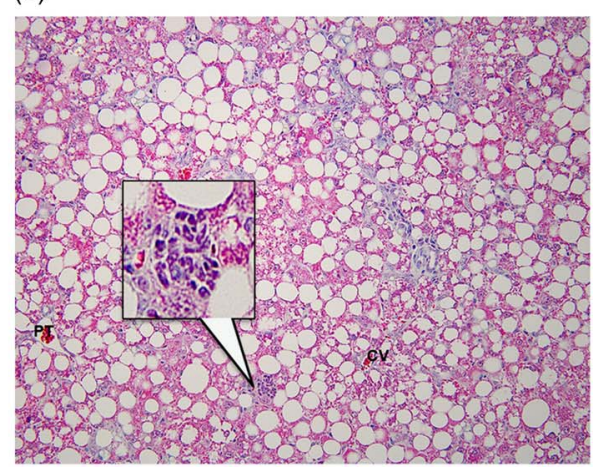

Fig. 2. Formalin-fixed, paraffin-embedded liver sections stained with Gomori's Trichrome from $d b / d b$ mice fed either a high-fat, high-sucrose diet or a methionine- and choline-deficient (MCD) diet. All images are at 20X magnification with insets at 40X magnification. (a) db/db mice fed a high-fat, high-sucrose diet for 12 weeks developed microvesicular steatosis. (b) $d b / d b$ mice fed a MCD diet for 3 weeks had macrovesicular steatosis and lobular inflammation. CV, central vein; PT, portal tract.

\section{Leptin deficient (ob/ob) and leptin receptor deficient (db/db)}

Numerous transgenic mouse models have been used to study obesity and related co-morbidities including the leptin-deficient $o b / o b$ mouse and the $d b / d b$ mouse that lacks functional leptin receptor signalling ${ }^{(46,51,52)}$. Shortly following weaning these mice become obese, insulin resistant and develop steatosis on a chow diet which can become exacerbated on a HFD ${ }^{(51)}$ (Fig. 2(a)). Although these mice readily develop steatosis they do not consistently progress to steatohepatitis without additional metabolic challenge ${ }^{(26)}$ (Fig. 2(b)). Following 10 weeks of high-saturated fat diet feeding in $d b / d b$ mice, half of the cohort developed NAFLD while the remaining mice progressed to $\mathrm{NASH}^{(26)}$. There was an inverse relationship between $d b / d b$ mice with $\mathrm{NASH}$ and levels of adiponectin, suggesting that hypoadiponectinaemia may be a critical component of the metabolic syndrome and the progression to $\mathrm{NASH}^{(26)}$. A thorough review of the variety of genetic models available to study NAFLD has been recently published ${ }^{(46)}$.

\section{$\mathrm{foz} / \mathrm{foz}$}

Similar to Alström syndrome in humans, foz/foz mice with a mutation in the Alms1 gene manifest a disease phenotype characterised by obesity and type 2 diabetes ${ }^{(53)}$. After $250 \mathrm{~d}$ of feeding a regular chow diet both male and female foz/foz mice develop significantly increased body fat mass compared with wild-type controls $^{(53)}$. Switching foz/foz mice onto a HFD for 24 weeks leads to an accelerated obesity phenotype and the development of steatohepatitis including elevated ALT levels, lobular inflammation, ballooning hepatocytes and increased expression of fibrosis genes type 1 collagen, $\alpha$-smooth muscle actin ( $\alpha$-SMA) and matrix metallopeptidase 9 (MMP-9) ${ }^{(54,55)}$. Interestingly, when foz/foz mice are switched back to a chow diet after 12 weeks of HFD feeding they have improved liver function, reduced blood glucose levels and increased adiponectin compared with foz/foz mice fed on $\mathrm{HFD}^{(54)}$. The reduced progression of steatohepatitis was linked to a shift in lipid metabolism including the redistribution of TAG storage in adipose tissue over the liver, reduced hepatic fatty acid uptake and macrophage activation favouring tissue recovery over fibrosis, demonstrating a key link between diet and the progression of $\mathrm{NASH}^{(54)}$.

\section{Murine models best for diet-induced non-alcoholic fatty liver disease}

When choosing an animal model to study NAFLD there are a number of characteristics that should be considered. Due to the association between adipose tissue inflammation, insulin resistance and steatosis, diets high in saturated fat are often used to model obesity, glucose intolerance and NAFLD in mice ${ }^{(56)}$. Depending on the primary aim of the study it is key to identify the differences in dietary models as HFD alone have been shown to lead to alterations in weight gain and insulin sensitivity compared with high-sucrose and high-fat diets, and highsucrose diets ${ }^{(57,58)}$. Comparison of diets ranging in fat from 30 to $60 \%$ demonstrated that a diet containing $>45 \%$ of energy from fat results in the greatest weight gain after 18 weeks of treatment in CD-1 mice ${ }^{(56)}$. Extrapolation of findings from HFD-fed mice must be used with caution as certain obesity-related diseases are more representative of the human condition than others ${ }^{(59)}$.

\section{High-fat diets}

Dietary treatment with a diet high in fat is one of the most common animal models to study obesity, insulin resistance and liver steatosis ${ }^{(60-65)}$. Often the diets are composed of $40-60 \%$ energy from fat that can be a derived from a combination of saturated fat, probably from lard and unsaturated fat in maize oil $^{(66)}$. One of the most critical factors when employing the HFD model is that a long duration of feeding, usually greater than 10 weeks, is required for the full effects of the diet to manifest into obesity, glucose intolerance and NAFLD ${ }^{(60-65)}$. While mice fed a HFD for over 10 weeks develop steatosis, a much longer duration exceeding 40 weeks of HFD feeding is required to initiate a mild degree of inflammation more closely representing $\mathrm{NASH}^{(67)}$.

In addition to increased fat mass and impaired glucose tolerance, long-term HFD feeding has also been linked to a number of defects including delayed fracture healing in mice $^{(68)}$. At 5 weeks following injury, mice on a HFD had reduced bone volume and biomechanically weaker repair 
(a)

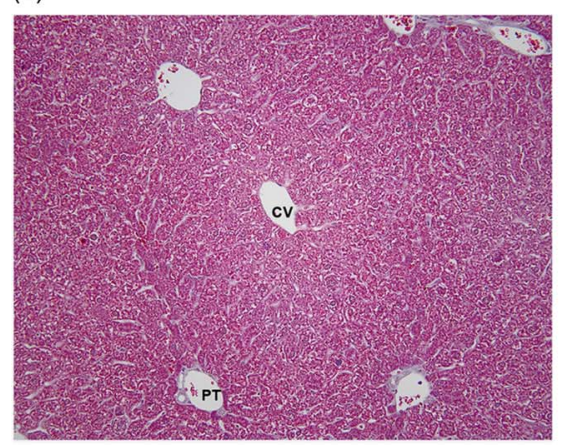

(b)

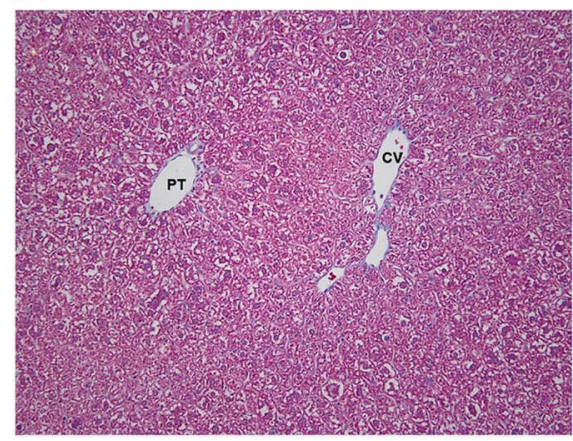

(c)

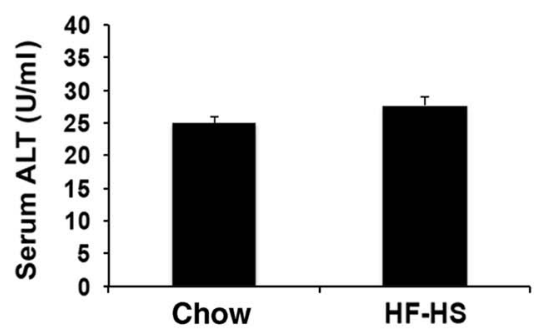

Fig. 3. Formalin-fixed, paraffin-embedded liver sections stained with Gomori's Trichrome from C57BL/6 mice fed either a standard chow diet or a high-fat, high-sucrose (HF-HS) diet. All images are at 20X magnification. (a) C57BL/6 mice fed a standard chow diet. (b) C57BL/6 fed a HF-HS diet for 10 weeks, demonstrating mild steatosis. (c) Serum alanine aminotransferase (ALT) levels of mice fed a standard chow diet or a HF-HS diet. Values are means, with standard errors represented by vertical bars. No statistical difference was detected $(P=0.14)$. CV, central vein; PT, portal tract.

compared with chow-fed mice ${ }^{(68)}$. It was proposed that this difference was due to an increased number of adipocytes at the fracture location and a subsequent loss of mesenchymalderived osteoblasts ${ }^{(68)}$. The mutually exclusive relationship between adipocyte formation and bone formation from mesenchymal stem cells continues to be evaluated ${ }^{(68)}$. Recently, HFD feeding for 8 weeks was also associated with reduced metabolic activity at the blood-brain barrier ${ }^{(69)}$. Analysis of HFD feeding for 10 weeks on the lymphatic system demonstrated a reduction in inguinal lymph node size, dysregulation of $\mathrm{T}$ cell migration, increased infiltration of $\mathrm{B}$ cells and macrophages and impaired lymphatic fluid transport ${ }^{(70)}$.

Sex and age differences between male and female C57B/Bl6 mice on HFD feeding have been reported ${ }^{(71)}$. Male mice fed HFD on average consume more energy and therefore weigh more than their chow-fed male controls as well as female mice fed HFD ${ }^{(71)}$. There was an increase in fat mass in both male and female groups fed HFD whereas no difference in fat-free mass $^{(71)}$. In addition, older-aged mice ( $>23$ weeks of age) were found to have greater variability in fat mass than young mice (8 weeks of age $)^{(71)}$. Future studies employing wild-type mice should consider how sex and age differences might affect the study outcome.

\section{'Westernised' diet}

Combination of a HFD with added fructose or sucrose is considered to be more reflective of a 'Westernised' diet and has been characterised ${ }^{(72,73)}$. After 16 weeks of feeding the American lifestyle-induced obesity syndrome (ALIOS) diet in male $\mathrm{C} 57 \mathrm{BL} / 6$ mice, increased weight gain, elevated serum glucose levels and higher ALT levels compared with chow-fed mice were observed $^{(72)}$. The ALIOS diet is composed of $45 \%$ of energy from fat as well as high-fructose maize syrup added in the drinking water. Mice fed the ALIOS diet develop macrovesicular steatosis in zones one and two and microvesicular steatosis predominately in zone three ${ }^{(72)}$. The development of NASH was limited to few inflammatory foci but there was increased expression of TNF- $\alpha$ within hepatocytes ${ }^{(72)}$. It has been observed that even after 24 weeks of HFD feeding plus addition of fructose in the drinking water $(5 \%, \mathrm{w} / \mathrm{v})$, approximately $30 \%$ of mice in the cohort resisted excessive weight gain and remained insulin sensitive ${ }^{(74)}$. This evidence suggests that 'Westernised' diet models should be fed for minimally 4 months and span upwards of 9 months ${ }^{(72)}$. In addition each cohort of mice should be stratified based on weight gain to identify mice that are prone to obesity compared with mice that remain lean following high-energy-diet feeding ${ }^{(74)}$. We have found that feeding male C57BL/6 mice a high-fat high-sucrose diet for 10 weeks results in mild steatosis and slightly elevated serum ALT levels (Fig. 3(a)-(c)).

\section{Carbohydrate-enriched diet}

Diets enriched with refined carbohydrates such as sucrose and fructose have been found to increase the susceptibility of mice to develop steatosis and insulin resistance similar to that of the human condition ${ }^{(75-79)}$. Mice fed a diet high in fat and refined carbohydrates developed hyperglycaemia and impaired glucose tolerance compared with HFD alone in C57BL/6 mice $^{(76)}$. Supplementing both male and female C57BL/6 mice with $30 \%$ fructose drinking water for 16 weeks led to greater weight gain in male mice but when normalised to liver weight, female mice were found to have a significantly greater liver: body-weight ratio ${ }^{(75)}$. Histological assessment of liver tissue demonstrated significant development of steatosis in both male and female mice; however, serum ALT and inflammation scores did not differ significantly between mice on the fructose solution compared with normal drinking water ${ }^{(75)}$. In addition, chronic fructose feeding led to increased portal endotoxin levels in both male and female mice, TNF $\alpha$ concentration; also Toll-like receptor adaptor molecule MyD88 expression was elevated in these groups ${ }^{(75)}$. Heightened changes in female mice over male mice was linked to decreased adiponectin levels in adipose tissue as well as increased levels of acutephase protein-1 in the liver and lower phosphorylation of $5^{\prime}$ AMP-activated protein kinase (AMPK) in the liver of female mice $^{(75)}$. A HFD along with the addition of fructose leads to substantial hepatic steatosis and represents a good model to study the initiation of NAFLD ${ }^{(75)}$. 
(a)

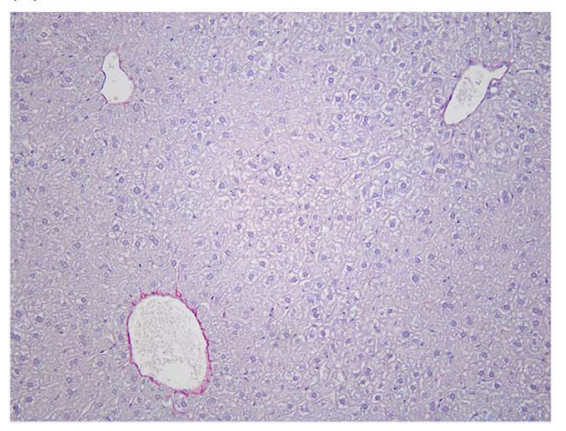

(b)

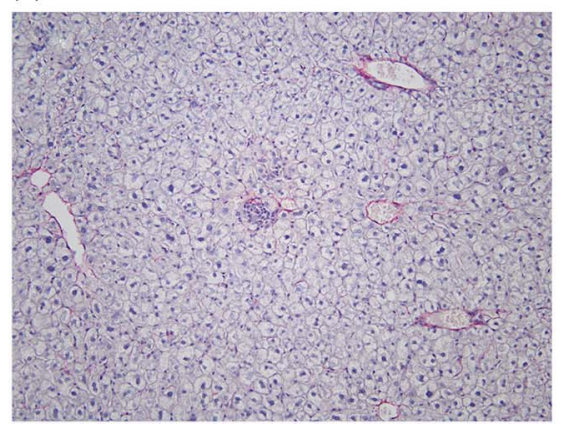

(c)

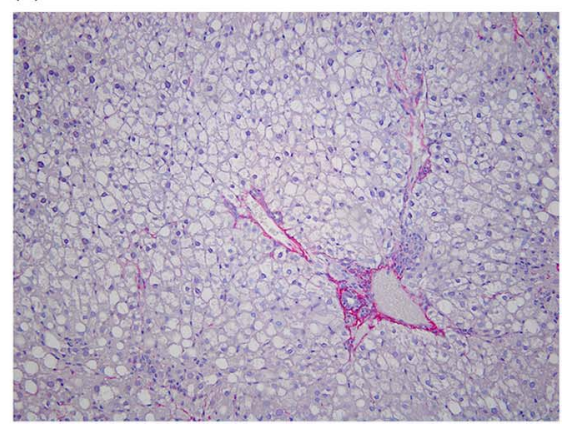

Fig. 4. Formalin-fixed, paraffin-embedded liver sections stained with Picrosirius Red from C57BL/6 mice fed either a standard chow diet or a methionine- and cholinedeficient (MCD) diet. All images are at 20X magnification. (a) C57BL/6 fed a standard chow diet. (b) C57BL/6 mice fed a MCD diet for 3 weeks. Mild fibrosis is evident forming around lipid-laden hepatocytes. (c) C57BL/6 mice fed a MCD diet for 6 weeks. Extended diet treatment results in the development of bridging fibrosis within the liver reflective of chronic inflammation and hepatocellular damage.

\section{Murine models best for diet-induced non-alcoholic steatohepatitis}

\section{Methionine- and choline-deficient diet}

Methionine and choline deficiency is a well-characterised dietary rodent model of $\mathrm{NASH}^{(80)}$. Choline is considered an essential nutrient that is required for cell membrane integrity, the neurotransmitter acetylcholine and synthesis of VLDL ${ }^{(15)}$. As well, choline deficiency has been linked to cellular apoptosis as evident by hepatocyte cell death when placed in a cholinedeficient medium. When choline deficiency is coupled with the lack of the essential amino acid methionine, mice develop steatosis and inflammation within 3 weeks and can progress to fibrosis with longer duration of feeding (6-8 weeks on average $)^{(81,82)}$ (Fig. 4(a)-(c)). A similar trend has been observed in patients receiving TPN ${ }^{(83)}$. Choline supplementation in TPN feeding was shown to improve liver biochemistry and should be added to long-term TPN to help prevent liver disease ${ }^{(83)}$. The pathogenesis of NASH in the methionine- and choline-deficient (MCD) diet model is thought to be due to impaired $\beta$-oxidation and heightened production of reactive oxygen species leading to oxidative stress. Development of NASH in this model has also been associated with the secretion of proinflammatory cytokines and chemokines and the subsequent recruitment of leucocytes into the liver ${ }^{(38)}$

Recently MCD diet feeding was associated with disrupted vascular architecture within the liver, suggesting that angiogenesis may be connected to the progression of liver fibrosis, cirrhosis and HCC. After 4 weeks of MCD diet feeding C57BL6/J mice had increased vascular endothelial growth factor (VEGF) in the liver and treatment with $\alpha$ VEGFR2 was able to reduce the degree of inflammation and hepatocellular ballooning after 8 weeks of MCD diet feeding ${ }^{(84)}$. The availability and ease of use makes the MCD diet model a valuable tool to investigate the role of inflammation during NASH. However, animals become cachectic and may lose up to $50 \%$ of their body weight, ameliorating any obese or insulin-resistant phenotype. We have found that following 3 weeks of MCD diet treatment, dietinduced obese mice develop steatohepatitis; however, their blood glucose levels normalise within the MCD diet feeding period (DT Reid, unpublished results). Recently a new diet combining choline deficiency with HFD was demonstrated to lead to hepatic steatosis, inflammation and fibrosis while the mice retained a stable body weight ${ }^{(85)}$. Further experimentation is required to characterise the metabolic status of this dietary treatment; however, this diet represents a novel approach to studying NASH in the context of the metabolic syndrome ${ }^{(85)}$.

Use of the $d b / d b$ mouse in combination with the MCD diet treatment has also been used as an attractive model of obesity, insulin resistance and $\mathrm{NASH}^{(86,87)}$. In this case insulin resistance is manifested by the hyperphagic behaviour of the mice while at the same time the MCD diet feeding induces inflammation within the liver. Following 4 weeks of diet, $d b / d b$ mice are susceptible to NASH and are found to have steatosis, inflammatory granulomas present in liver tissue and elevated ALT levels ${ }^{(88)}$. However, we have found that after only 3 weeks of MCD diet feeding, $d b / d b$ mice lose approximately $15 \%$ of their original body weight and have significantly lower glucose AUC compared with HFD-fed $d b / d b$ mice following a 90 min oral glucose tolerance test. This suggests that although $d b / d b$ mice develop NASH on the MCD diet, their insulin sensitivity has improved and may no longer represent a model of the metabolic syndrome.

\section{Extended duration of high-fat diets}

Long-term HFD studies have become a popular model to study the development of NASH. Use of a 'fast food' diet rich in saturated fat and fructose in $\mathrm{C} 57 \mathrm{BL} / 6$ mice for 25 weeks resulted in greater body weight, elevated aminotransferase levels and homeostatic model assessment of insulin resistance (HOMA-IR) scores compared with chow-fed mice ${ }^{(89)}$. This diet led to steatosis and evidence of ballooning hepatocytes. As well, bridging fibrosis was evident following 25 weeks of HFD feeding. The combination of high fat plus fructose ${ }^{(90)}$ in this diet was central to the development of NASH within these mice. Compared with the fast food diet, HF diet-fed mice did not develop fibrosis and had substantially less inflammation and hepatocyte ballooning even after 25 weeks of $\mathrm{HF}$ diet treatment. Histological comparison between MCD diet and HF diet feeding demonstrates similar disease progression; however, HF diet treatment requires longer treatment duration and on 
average leads to less severe NASH. Vitamin D deficiency has been found to accelerate NASH in a HFD model ${ }^{(22)}$. This phenotype was attributed to the loss of bile acid uptake through the ileal apical Na-dependent bile acid cotransporter (iABST) and increased de novo lipogenesis leading to heightened inflammation and exacerbation to steatohepatitis ${ }^{(22)}$.

\section{High-cholesterol and-cholic acid diets}

Similar to the HFD model, diets high in cholesterol and cholic acid are considered a more atherogenic diet and have been developed as a model of $\mathrm{NASH}^{(91)}$. A period of 30 weeks of high-fat/high-cholesterol feeding (15\%, $1 \% \quad(\mathrm{w} / \mathrm{w})$, respectively) resulted in weight gain and steatohepatitis including elevated ALT levels, steatosis, lobular inflammation and perisinusoidal fibrosis ${ }^{(92)}$. Critically, the development of NASH was dependent on the combined high-fat and highcholesterol feeding as neither a HFD or high-cholesterol diet alone led to steatohepatitis ${ }^{(92)}$. A combination of high-fat, high-cholesterol and high-fructose diet resulted in hepatic steatosis, exacerbated TNF-related apoptosis-inducing ligand (TRAIL)-mediated liver injury, increased expression of proinflammatory genes and elevated Mac-2 staining in the liver after 12 weeks of treatment ${ }^{(93)}$. Further study of the altered metabolism in mice fed this diet is required to fully elucidate the disease model.

\section{Development of hepatocellular carcinoma during non-alcoholic fatty liver disease}

Mirroring the increasing rates of obesity, type 2 diabetes and NAFLD is the increased incidence of $\mathrm{HCC}^{(94)}$. NAFLD is now considered a risk factor for the development of HCC as well as both cirrhotic and non-cirrhotic $\mathrm{NASH}^{(94-96)}$. Animal models have been used to study HCC in the context of NAFLD ${ }^{(94)}$. In one case male neonatal mice exposed to streptozotocin followed HFD feeding develop insulin resistance and hepatic steatosis that can progress to fibrosis and HCC (STAM model) ${ }^{(97)}$. NASH-related HCC in the absence of cirrhosis has also been described in patient populations; however, animal models to investigate this relationship have not been reported $^{(98)}$.

\section{Conclusion}

Multiple rodent models can be used to study the pathogenesis of NAFLD and NASH in the context of obesity. In particular, long-term feeding of certain dietary components such as saturated fat, cholesterol and fructose can lead to steatosis as well as mild inflammation and hepatocyte damage. Diets deficient in methionine and choline provide a robust model to study leucocyte infiltration during NASH. However, particular care should be taken when selecting an animal model to study NAFLD and NASH as current available models do not represent the full disease spectrum. Continued evolution of animal models to investigate NAFLD and NASH is required.

\section{Acknowledgements}

The present review was supported by a Natural Sciences and Engineering Research Council of Canada (NSERC) fellowship (to D. T. R.) and a Canadian Institutes of Health Research (CIHR) Signature Initiative Team grant (to B. E.).

D. T. R. and B. E. have no financial disclosures relating to this body of work.

\section{References}

1. Caballero B (2007) The global epidemic of obesity: an overview. Epidemiol Rev 29, 1-5.

2. Rezaianzadeh A, Namayandeh SM \& Sadr SM (2012) National Cholesterol Education Program Adult Treatment Panel III versus International Diabetic Federation Definition of metabolic syndrome, which one is associated with diabetes mellitus and coronary artery disease? Int J Prev Med 3, 552-558.

3. Boyle JP, Thompson TJ, Gregg EW, et al. (2010) Projection of the year 2050 burden of diabetes in the US adult population: dynamic modeling of incidence, mortality, and prediabetes prevalence. Popul Health Metr 8, 29.

4. Hamaguchi M, Takeda N, Kojima T, et al. (2012) Identification of individuals with non-alcoholic fatty liver disease by the diagnostic criteria for the metabolic syndrome. World $J$ Gastroenterol 18, 1508-1516.

5. Levene AP \& Goldin RD (2012) The epidemiology, pathogenesis and histopathology of fatty liver disease. Histopatbology 61, 141-152.

6. Michelotti GA, Machado MV \& Diehl AM (2013) NAFLD, NASH and liver cancer. Nat Rev Gastroenterol Hepatol 10, 656-665.

7. Bellentani S \& Tiribelli C (2001) The spectrum of liver disease in the general population: lesson from the Dionysos study. J Hepatol 35, 531-537.

8. Yasui K, Hashimoto E, Komorizono Y, et al. (2011) Characteristics of patients with nonalcoholic steatohepatitis who develop hepatocellular carcinoma. Clin Gastroenterol Hepatol 9, 428-433, e50.

9. Engl J, Sturm W, Sandhofer A, et al. (2008) Effect of pronounced weight loss on visceral fat, liver steatosis and adiponectin isoforms. Eur J Clin Invest 38, 238-244.

10. Milic S \& Stimac D (2012) Nonalcoholic fatty liver disease/ steatohepatitis: epidemiology, pathogenesis, clinical presentation and treatment. Dig Dis 30, 158-162.

11. Pacana T \& Fuchs M (2012) The cardiovascular link to nonalcoholic fatty liver disease: a critical analysis. Clin Liver Dis 16, 599-613.

12. Bugianesi E, Moscatiello S, Ciaravella MF, et al. (2010) Insulin resistance in nonalcoholic fatty liver disease. Curr Pharm Des 16, 1941-1951.

13. Tilg H \& Moschen AR (2008) Insulin resistance, inflammation, and non-alcoholic fatty liver disease. Trends Endocrinol Metab 19, 371-379.

14. Larrain S \& Rinella ME (2012) A myriad of pathways to NASH. Clin Liver Dis 16, 525-548.

15. Buchman AL (2009) The addition of choline to parenteral nutrition. Gastroenterology 137, Suppl. 5, S119-S128.

16. Petta S, Vanni E, Bugianesi E, et al. (2015) PNPLA3 rs738409 $\mathrm{I} 748 \mathrm{M}$ is associated with steatohepatitis in 434 non-obese subjects with hepatitis C. Aliment Pharmacol Ther 41, 939-948.

17. Richmond TK, Thurston I, Sonneville K, et al. (2015) Racial/ ethnic differences in accuracy of body mass index reporting in a diverse cohort of young adults. Int J Obes (Lond) 39, 546-548. 
18. Nigam P, Misra A \& Colles SL (2013) Comparison of DEXA-derived body fat measurement to two race-specific bioelectrical impedance equations in healthy Indians. Diabetes Metab Syndr 7, 72-77.

19. Jitnarin N, Poston WS, Haddock CK, et al. (2014) Accuracy of body mass index-defined obesity status in US firefighters. Saf Health Work 5, 161-164.

20. Machado MV, Michelotti GA, Xie G, et al. (2015) Mouse models of diet-induced nonalcoholic steatohepatitis reproduce the heterogeneity of the human disease. PLOS ONE $\mathbf{1 0}$, e0127991.

21. Ludwig J, Viggiano TR, McGill DB, et al. (1980) Nonalcoholic steatohepatitis: Mayo Clinic experiences with a hitherto unnamed disease. Mayo Clin Proc 55, 434-438.

22. Kong M, Zhu L, Bai L, et al. (2014) Vitamin D deficiency promotes nonalcoholic steatohepatitis through impaired enterohepatic circulation in animal model. Am J Physiol Gastrointest Liver Physiol 307, G883-G893.

23. Donnelly KL, Smith CI, Schwarzenberg SJ, et al. (2005) Sources of fatty acids stored in liver and secreted via lipoproteins in patients with nonalcoholic fatty liver disease. J Clin Invest 115, 1343-1351.

24. Lottenberg AM, Afonso Mda S, Lavrador MS, et al. (2012) The role of dietary fatty acids in the pathology of metabolic syndrome. J Nutr Biochem 23, 1027-1040.

25. Das SK \& Balakrishnan V (2011) Role of cytokines in the pathogenesis of non-alcoholic fatty liver disease. Indian J Clin Biochem 26, 202-209.

26. Handa P, Maliken BD, Nelson JE, et al. (2014) Reduced adiponectin signaling due to weight gain results in nonalcoholic steatohepatitis through impaired mitochondrial biogenesis. Hepatology 60, 133-145.

27. Kim KH, Jeong YT, Oh H, et al. (2013) Autophagy deficiency leads to protection from obesity and insulin resistance by inducing Fgf21 as a mitokine. Nat Med 19, 83-92.

28. Kwanten WJ, Martinet W, Michielsen PP, et al. (2014) Role of autophagy in the pathophysiology of nonalcoholic fatty liver disease: a controversial issue. World I Gastroenterol 20, $7325-7338$.

29. Tuyama AC \& Chang CY (2012) Non-alcoholic fatty liver disease. J Diabetes 4, 266-280.

30. Neuschwander-Tetri BA, Clark JM, Bass NM, et al. (2010) Clinical, laboratory and histological associations in adults with nonalcoholic fatty liver disease. Hepatology $\mathbf{5 2}$, 913-924.

31. Kanuri G, Landmann M, Priebs J, et al. (2015) Moderate alcohol consumption diminishes the development of non-alcoholic fatty liver disease (NAFLD) in $o b / o b$ mice. Eur J Nutr (epublication ahead of print version 24 May 2015).

32. Wieckowska A \& Feldstein AE (2008) Diagnosis of nonalcoholic fatty liver disease: invasive versus noninvasive. Semin Liver Dis 28, 386-395.

33. Day CP \& James OF (1998) Steatohepatitis: a tale of two "hits"? Gastroenterology 114, 842-845.

34. Musso G, Gambino R \& Cassader M (2013) Cholesterol metabolism and the pathogenesis of non-alcoholic steatohepatitis. Prog Lipid Res 52, 175-191.

35. Volynets V, Kuper MA, Strahl S, et al. (2012) Nutrition, intestinal permeability, and blood ethanol levels are altered in patients with nonalcoholic fatty liver disease (NAFLD). Dig Dis Sci 57, 1932-1941.

36. Day CP \& James OF (1998) Hepatic steatosis: innocent bystander or guilty party? Hepatology 27, 1463-1466.

37. Rahimi RS \& Landaverde C (2013) Nonalcoholic fatty liver disease and the metabolic syndrome: clinical implications and treatment. Nutr Clin Pract 28, 40-51.
38. Farrell GC, van Rooyen D, Gan L, et al. (2012) NASH is an inflammatory disorder: pathogenic, prognostic and therapeutic implications. Gut Liver 6, 149-171.

39. Pagadala MR \& McCullough AJ (2012) The relevance of liver histology to predicting clinically meaningful outcomes in nonalcoholic steatohepatitis. Clin Liver Dis 16, 487-504.

40. Waller-Evans H, Hue C, Fearnside J, et al. (2013) Nutrigenomics of high fat diet induced obesity in mice suggests relationships between susceptibility to fatty liver disease and the proteasome. PLOS ONE $\mathbf{8}$, e82825.

41. Montgomery MK, Hallahan NL, Brown SH, et al. (2013) Mouse strain-dependent variation in obesity and glucose homeostasis in response to high-fat feeding. Diabetologia 56, 1129-1139.

42. Nagao M, Asai A, Inaba W, et al. (2014) Characterization of pancreatic islets in two selectively bred mouse lines with different susceptibilities to high-fat diet-induced glucose intolerance. PLOS ONE 9, e84725.

43. Sims EK, Hatanaka M, Morris DL, et al. (2013) Divergent compensatory responses to high-fat diet between C57BL6/J and C57BLKS/J inbred mouse strains. Am J Physiol Endocrinol Metab 305, E1495-E1511.

44. Heiker JT, Kunath A, Kosacka J, et al. (2014) Identification of genetic loci associated with different responses to high-fat diet-induced obesity in $\mathrm{C} 57 \mathrm{BL} / 6 \mathrm{~N}$ and $\mathrm{C} 57 \mathrm{BL} / 6 \mathrm{~J}$ substrains. Physiol Genomics 46, 377-384.

45. Podrini C, Cambridge EL, Lelliott CJ, et al. (2013) High-fat feeding rapidly induces obesity and lipid derangements in C57BL/6N mice. Mamm Genome 24, 240-251.

46. Nagarajan P, Mahesh Kumar MJ, Venkatesan R, et al. (2012) Genetically modified mouse models for the study of nonalcoholic fatty liver disease. World J Gastroenterol 18, $1141-1153$

47. d Oliveira RB, Carvalho CP, Polo CC, et al. (2014) Impaired compensatory $\beta$-cell function and growth in response to high-fat diet in LDL receptor knockout mice. Int J Exp Pathol 95, 296-308.

48. Bojic LA, Burke AC, Chhoker SS, et al. (2014) Peroxisome proliferator-activated receptor $\delta$ agonist GW1516 attenuates diet-induced aortic inflammation, insulin resistance, and atherosclerosis in low-density lipoprotein receptor knockout mice. Arterioscler Thromb Vasc Biol 34, 52-60.

49. Depner CM, Traber MG, Bobe G, et al. (2013) A metabolomic analysis of omega-3 fatty acid-mediated attenuation of Western diet-induced nonalcoholic steatohepatitis in $L D L R^{-/}$mice. PLOS ONE 8, e83756.

50. Subramanian S, Goodspeed L, Wang S, et al. (2011) Dietary cholesterol exacerbates hepatic steatosis and inflammation in obese LDL receptor-deficient mice. J Lipid Res 52, 1626-1635.

51. Trak-Smayra V, Paradis V, Massart J, et al. (2011) Pathology of the liver in obese and diabetic $o b / o b$ and $d b / d b$ mice fed a standard or high-calorie diet. Int J Exp Pathol 92, 413-421.

52. Tamura Y, Sugimoto M, Murayama T, et al. (2008) Inhibition of CCR2 ameliorates insulin resistance and hepatic steatosis in db/db mice. Arterioscler Thromb Vasc Biol 28, 2195-2201.

53. Arsov T, Silva DG, O'Bryan MK, et al. (2006) Fat aussie - a new Alström syndrome mouse showing a critical role for ALMS1 in obesity, diabetes, and spermatogenesis. Mol Endocrinol 20, 1610-1622.

54. Larter CZ, Yeh MM, Haigh WG, et al. (2013) Dietary modification dampens liver inflammation and fibrosis in obesity-related fatty liver disease. Obesity (Silver Spring) 21, 1189-1199.

55. Van Rooyen DM, Larter CZ, Haigh WG, et al. (2011) Hepatic free cholesterol accumulates in obese, diabetic mice and causes nonalcoholic steatohepatitis. Gastroenterology 141, 1393-1403, 1403.e1-1403.e5. 
56. Carpenter KC, Strohacker K \& McFarlin BK (2013) Considerations to maximize fat mass gain in a mouse model of diet-induced weight gain. Lab Anim 47, 266-273.

57. Omar B, Pacini G \& Ahrén B (2012) Differential development of glucose intolerance and pancreatic islet adaptation in multiple diet induced obesity models. Nutrients $\mathbf{4}, 1367-1381$.

58. Pyndt Jorgensen B, Hansen JT, Krych L, et al. (2014) A possible link between food and mood: dietary impact on gut microbiota and behavior in BALB/c mice. PLOS ONE 9, e103398.

59. Brainard RE, Watson LJ, Demartino AM, et al. (2013) High fat feeding in mice is insufficient to induce cardiac dysfunction and does not exacerbate heart failure. PLOS ONE 8, e83174.

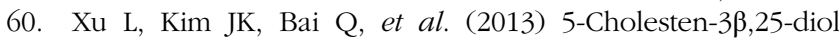
3-sulfate decreases lipid accumulation in diet-induced nonalcoholic fatty liver disease mouse model. Mol Pharmacol 83, 648-658.

61. Corbin JA, Bhaskar V, Goldfine ID, et al. (2014) Improved glucose metabolism in vitro and in vivo by an allosteric monoclonal antibody that increases insulin receptor binding affinity. PLOS ONE 9, e88684.

62. Carpene C, Gomez-Zorita S, Gupta R, et al. (2014) Combination of low dose of the anti-adipogenic agents resveratrol and phenelzine in drinking water is not sufficient to prevent obesity in very-high-fat diet-fed mice. Eur J Nutr 53, 1625-1635.

63. Clemmensen C, Smajilovic S, Madsen AN, et al. (2013) Increased susceptibility to diet-induced obesity in GPRC6A receptor knockout mice. J Endocrinol 217, 151-160.

64. Noyan-Ashraf MH, Shikatani EA, Schuiki I, et al. (2013) A glucagon-like peptide-1 analog reverses the molecular pathology and cardiac dysfunction of a mouse model of obesity. Circulation 127, 74-85.

65. Galloway CA, Lee H, Brookes PS, et al. (2014) Decreasing mitochondrial fission alleviates hepatic steatosis in a murine model of nonalcoholic fatty liver disease. Am J Physiol Gastrointest Liver Physiol 307, G632-G641.

66. Lafarge JC, Pini M, Pelloux V, et al. (2014) Cathepsin S inhibition lowers blood glucose levels in mice. Diabetologia $\mathbf{5 7}$, $1674-1683$.

67. Hennig EE, Mikula M, Goryca K, et al. (2014) Extracellular matrix and cytochrome $\mathrm{P} 450$ gene expression can distinguish steatohepatitis from steatosis in mice. J Cell Mol Med 18, 1762-1772.

68. Brown ML, Yukata K, Farnsworth CW, et al. (2014) Delayed fracture healing and increased callus adiposity in a C57BL/6J murine model of obesity-associated type 2 diabetes mellitus. PLOS ONE 9, e99656.

69. Ouyang S, Hsuchou H, Kastin AJ, et al. (2014) Diet-induced obesity suppresses expression of many proteins at the blood-brain barrier. J Cereb Blood Flow Metab 34, 43-51.

70. Weitman ES, Aschen SZ, Farias-Eisner G, et al. (2013) Obesity impairs lymphatic fluid transport and dendritic cell migration to lymph nodes. PLOS ONE 8, e70703.

71. Yang Y, Smith DL Jr, Keating KD, et al. (2014) Variations in body weight, food intake, and body composition after long-term high-fat diet feeding in C57BL/6J mice. Obesity (Silver Spring) 22, 2147-2155.

72. Tetri LH, Basaranoglu M, Brunt EM, et al. (2008) Severe NAFLD with hepatic necroinflammatory changes in mice fed trans fats and a high-fructose corn syrup equivalent. Am J Physiol Gastrointest Liver Physiol 295, G987-G995.

73. Yang ZH, Miyahara H, Takeo J, et al. (2012) Diet high in fat and sucrose induces rapid onset of obesity-related metabolic syndrome partly through rapid response of genes involved in lipogenesis, insulin signalling and inflammation in mice. Diabetol Metab Syndr 4, 32 .

74. Tan JT, McLennan SV, Williams PF, et al. (2013) Connective tissue growth factor/CCN-2 is upregulated in epididymal and subcutaneous fat depots in a dietary-induced obesity model. Am J Physiol Endocrinol Metab 304, E1291-E1302.

75. Spruss A, Henkel J, Kanuri G, et al. (2012) Female mice are more susceptible to nonalcoholic fatty liver disease: sexspecific regulation of the hepatic AMP-activated protein kinase-plasminogen activator inhibitor 1 cascade, but not the hepatic endotoxin response. Mol Med 18, 1346-1355.

76. Hodgson K, Govan B, Ketheesan N, et al. (2013) Dietary composition of carbohydrates contributes to the development of experimental type 2 diabetes. Endocrine 43, 447-451.

77. Ritze Y, Bardos G, Hubert A, et al. (2014) Effect of tryptophan supplementation on diet-induced non-alcoholic fatty liver disease in mice. Br J Nutr 112, 1-7.

78. Perez-Martinez L, Perez-Matute P, Aguilera-Lizarraga J, et al. (2014) Maraviroc, a CCR5 antagonist, ameliorates the development of hepatic steatosis in a mouse model of nonalcoholic fatty liver disease (NAFLD). J Antimicrob Chemother 69, 1903-1910.

79. Oliveira MC, Menezes-Garcia Z, Henriques MC, et al. (2013) Acute and sustained inflammation and metabolic dysfunction induced by high refined carbohydrate-containing diet in mice. Obesity (Silver Spring) 21, E396-E406.

80. Heinrichs D, Berres ML, Coeuru M, et al. (2014) Protective role of macrophage migration inhibitory factor in nonalcoholic steatohepatitis. FASEB J 28, 5136-5147.

81. Weltman MD, Farrell GC \& Liddle C (1996) Increased hepatocyte CYP2E1 expression in a rat nutritional model of hepatic steatosis with inflammation. Gastroenterology 111, 1645-1653.

82. Bellafante E, Murzilli S, Salvatore L, et al. (2013) Hepaticspecific activation of peroxisome proliferator-activated receptor $\gamma$ coactivator- $1 \beta$ protects against steatohepatitis. Hepatology $\mathbf{5 7}$, 1343-1356.

83. Buchman AL, Ament ME, Sohel M, et al. (2001) Choline deficiency causes reversible hepatic abnormalities in patients receiving parenteral nutrition: proof of a human choline requirement: a placebo-controlled trial. I Parenter Enteral Nutr 25, 260-268.

84. Coulon S, Legry V, Heindryckx F, et al. (2013) Role of vascular endothelial growth factor in the pathophysiology of nonalcoholic steatohepatitis in two rodent models. Hepatology 57, 1793-1805.

85. Matsumoto M, Hada N, Sakamaki Y, et al. (2013) An improved mouse model that rapidly develops fibrosis in non-alcoholic steatohepatitis. Int J Exp Pathol 94, 93-103.

86. Sahai A, Malladi P, Pan X, et al. (2004) Obese and diabetic $d b / d b$ mice develop marked liver fibrosis in a model of nonalcoholic steatohepatitis: role of short-form leptin receptors and osteopontin. Am J Physiol Gastrointest Liver Physiol 287, G1035-G1043.

87. Rinella ME, Elias MS, Smolak RR, et al. (2008) Mechanisms of hepatic steatosis in mice fed a lipogenic methionine cholinedeficient diet. J Lipid Res 49, 1068-1076.

88. Salamone F, Galvano F, Marino Gammazza A, et al. (2012) Silibinin improves hepatic and myocardial injury in mice with nonalcoholic steatohepatitis. Dig Liver Dis 44, 334-342.

89. Charlton M, Krishnan A, Viker K, et al. (2011) Fast food diet mouse: novel small animal model of NASH with ballooning, progressive fibrosis, and high physiological fidelity to the human condition. Am J Physiol Gastrointest Liver Physiol 301, G825-G834.

90. Fujii H, Enomoto M, Fukushima W, et al. (2009) Noninvasive laboratory tests proposed for predicting cirrhosis in patients 
with chronic hepatitis $\mathrm{C}$ are also useful in patients with non-alcoholic steatohepatitis. J Gastroenterol 44, 608-614.

91. Matsuzawa N, Takamura T, Kurita S, et al. (2007) Lipidinduced oxidative stress causes steatohepatitis in mice fed an atherogenic diet. Hepatology 46, 1392-1403.

92. Savard C, Tartaglione EV, Kuver R, et al. (2013) Synergistic interaction of dietary cholesterol and dietary fat in inducing experimental steatohepatitis. Hepatology 57, 81-92.

93. Hirsova P, Ibrahim SH, Bronk SF, et al. (2013) Vismodegib suppresses TRAIL-mediated liver injury in a mouse model of nonalcoholic steatohepatitis. PLOS ONE $\mathbf{8}$, e70599.

94. Streba LA, Vere CC, Rogoveanu I, et al. (2015) Nonalcoholic fatty liver disease, metabolic risk factors, and hepatocellular carcinoma: an open question. World J Gastroenterol 21, 4103-4110.

95. Torres DM \& Harrison SA (2012) Nonalcoholic steatohepatitis and noncirrhotic hepatocellular carcinoma: fertile soil. Semin Liver Dis 32, 30-38.

96. Alexander J, Torbenson M, Wu TT, et al. (2013) Non-alcoholic fatty liver disease contributes to hepatocarcinogenesis in noncirrhotic liver: a clinical and pathological study.J Gastroenterol Hepatol 28, 848-854.

97. Fujii H \& Kawada N (2012) Inflammation and fibrogenesis in steatohepatitis. J Gastroenterol 47, 215-225.

98. Kawada N, Imanaka K, Kawaguchi T, et al. (2009) Hepatocellular carcinoma arising from non-cirrhotic nonalcoholic steatohepatitis. J Gastroenterol 44, 1190-1194. 